\title{
Discrepancies Between the Labels of Originator and Generic Pharmaceutical Products: Implications for Patient Safety
}

\author{
Alexandra Thoenes $^{1} \cdot$ Luca Cariolato $^{1} \cdot$ Julian Spierings $^{2} \cdot$ Alexis Pinçon $^{1}$
}

Published online: 22 April 2020

(c) The Author(s) 2020

\begin{abstract}
Background All drug marketing authorization holders have the legal obligation to collect data on the use of the products they market and to keep the labels of those products updated. As demonstrated by previous studies, many generic products have labels that are discrepant from the labels of their reference (originator) products. This fact may cause inconsistent messages to be disseminated to healthcare professionals and patients for the same active ingredient.

Objective These potential label discrepancies led us to investigate the degree of difference between labels for generic and originator products, the possible consequences of this discrepancy for patients, and its implications for risk minimization.

Products and Methods Drugs from different Anatomical Therapeutic Chemical classes were randomly selected from the Electronic Medicines Compendium. For each drug, the consistency and discrepancies between the summaries of product characteristics (SmPCs) for originator and generic products were analyzed for each safety-relevant section of the SmPC separately as well as across all of its sections. The percentile distribution of discrepancy classifications was calculated. The same method was applied when determining the potential impact of label discrepencies on patients.

Results Among the 50 drugs selected initially, 31 were eligible for the study and were analyzed further. Of those 31 drugs, $13(41.9 \%)$ presented critical label differences between originator and generic products, $6(19.4 \%)$ showed major label differences, 7 (22.6\%) exhibited minor label differences, and 5 (16.1\%) showed very minor label differences. Over $60 \%$ of the selected drugs $(19,61.3 \%)$ presented important (critical, major) label differences between originator and generic products. None of the selected drugs had fully aligned labels of originator and generic products. Label misalignments that could potentially have a fatal or life-threatening impact on the patient were observed for $4(12.9 \%)$ of the selected drugs. Label misalignments that could have a severe patient outcome were noted for 11 (35.5\%) of the selected drugs, and label misalignments that could have a medium impact on the patient were seen for $6(19.35 \%)$ of the selected drugs. The label misalignments observed for $10(32.25 \%)$ of the selected drugs would potentially lead to only a minor or no effect on the patient. Almost half $(15,48.4 \%)$ of the selected drugs presented label misalignments that could have a critical (fatal, lifethreatening, severe) influence on the patient.

Conclusions In this sample, SmPC alignment between generic and originator medicinal products was found to be inefficient for established drugs, and could lead to the diffusion of discrepant messages to healthcare professionals and patients. In order to address this SmPC alignment problem, health authorities such as the EMA and the FDA must conduct retrospective analyses of all drugs on the market as a first step towards realigning labels. These analyses could be performed during the evaluation of aggregate reports.
\end{abstract}

\section{Introduction}

The label of a medicinal product plays an essential role in drug safety and risk minimization, as it is the primary source

Alexandra Thoenes

alexandra.thoenes@ubc.com

1 United BioSource LLC (UBC), Vernier, Geneva, Switzerland

2 Vifor Pharma, Meyrin, Geneva, Switzerland of information for healthcare professionals (HCPs [1]). In the European Union (EU), the reference safety information (RSI) for any medicinal product is the summary of product characteristics (SmPC), which is approved as part of the marketing authorization. The SmPC is aimed at HCPs 


\section{Key Points}

There are important differences between the labels for products based on the same active substance.

These differences in the safety information given to healthcare professionals for such products can be misleading and misinterpreted.

Patient safety may be compromised by these discrepancies between product labels.

Regulatory authorities should retrospectively align product labels during routine evaluations of aggregate reports.

specifically, informing them of the essential characteristics of each product and its safety profile, including contraindications, warnings, and adverse reactions. During the remainder of the lifecycle of a product, the SmPC is continually updated when new information emerges, notably due to the detection of new safety signals [2].

All drug marketing authorization holders (MAHs) have the legal obligation to collect data on the use of the products they market and to keep the labels of those products updated. Additionally, regulatory authorities ensure that any new safety information is appropriately described [3, 4].

MAHs that develop products with novel active ingredients (i.e., originators) should have access to considerably more clinical and preclinical data on those products than MAHs that produce generic versions of them. Also, the originators should have more extensive case databases, as they have more postmarketing experience than the companies that produce generic drugs. For this reason, originators are expected to maintain more comprehensive drug safety profiles than generic companies.

In the US, regulations currently require the labeling of generic medicines to be identical to their originator equivalents. However, in reality, the vast majority of generic drugs have labels that are discrepant from the reference (originator) drugs [5]. Directive 2004/27/EC of the European Commission [6] states that the Co-ordination Group for Mutual Recognition and Decentralised Procedures-Human (CMDh) is obliged to provide an annual list of medicinal products for which a harmonized SmPC should be drawn up. Despite this, the harmonization of many established drugs (those that have been on the market for 15 or more years) is inadequate. This fact causes inconsistent messages to be disseminated to HCPs and patients regarding products with the same active ingredient.

These potential label discrepancies between products led us to investigate the degree of difference between labels for products with the same active ingredient, the possible consequences of this difference for patients, and its implications for risk minimization.

\section{Methods}

\subsection{Data Source}

This comparative observational study was conducted between January and April 2019.

This study focused on drugs that were approved in the United Kingdom (UK) and had an international date of birth (IBD) between August 1945 and August 2004. This period was chosen to ensure that the drugs selected would be well established on the market and would be mature enough to have several generics available. The UK was taken as the country of reference because it was in the EU, where adverse drug reaction information is presented in SmPCs [7]. In the $\mathrm{UK}$, these SmPCs are publicly available on the Electronic Medicines Compendium (eMC [8]), which contains up-todate information concerning medicines licensed for use in the UK. The eMC has more than 10,600 documents, all of which have been verified and approved by either the Medicines and Healthcare Products Regulatory Agency (MHRA) or the European Medicines Agency (EMA), who license these medications.

Drugs belonging to different Anatomical Therapeutic Chemical (ATC [9]) classes were randomly selected from marketed established brands based on the active ingredients listed on the eMC. Randomization was performed using the RAND function in Microsoft ${ }^{\circledR}$ Excel $^{\circledR}$ 2016. A drug was eligible for the analysis if at least two marketed versions of it (i.e., two products) were available on the eMC. Drugs with nontherapeutic indications (e.g., vaccines and contrast agents) as well as medical devices were excluded.

\subsection{Determining Reference Products}

For this study, the SmPCs for the originator products were considered to be reference SmPCs. A request was made to the MHRA on 10 April 2019 to identify the originator products for the drugs selected for this study. A list containing the originator products for 24 of the analyzed drugs was provided. For the remaining seven drugs, the reference product was considered to be the product with the oldest marketing authorization date.

\subsection{Data Collection}

The safety information in Sections 4.3 (Contraindications), 4.4 (Special Warnings and Precautions), 4.8 (Undesirable Effects), and 4.9 (Overdose) of the SmPC for each product 
was extracted manually. Products with the same formulation but not necessarily the same dosage were compared to the originator product where available. A maximum of five SmPCs from different MAHs were analyzed for each drug. When more than five products were recovered for a drug, the superfluous products were selected at random (using the RAND function in Microsoft ${ }^{\circledR}$ Excel $^{\circledR}$ 2016) and then discarded.

\subsection{Category Assignment and Classification}

Two classification steps were performed for each drug: the first was based on the degree of difference between the SmPCs of originator and generic products, whereas the second was based on the potential impact of that SmPC difference on patients.

In the first step, each drug was classified into one of five categories (Table 1) based on the degree of difference between its SmPCs.

In the second classification step, the potential impact of any SmPC discrepancy on patients was assessed and categorized as shown in Table 2.

Two physicians independently reviewed the SmPC discrepancies and determined the potential impact on the patient. If there was disagreement about the categorization of the drug after examination, the two physicians met to adopt a common position.

\subsection{Literature Search}

A literature search was performed to verify if the inconsistent information in the reference RSI was supported by the published scientific literature.

Embase (1947 to June 2019) and MEDLINE (1951 to 24 June 2019) database searches were conducted on 24 June 2019. The MEDLINE database was searched using PubMed.

The following search terms were used:

- "Event"

- AND "Product."

When too many hits were recorded $(>50)$, the search was refined to

- "Event"

- AND "Product"

- AND "adverse event" OR "adverse reaction" OR "side effect."

Levels of evidence for each article were examined using the Oxford Center for Evidence-Based Medicine (OCEBM [11]) classification.
Table 1 Categories for the first classification step (based on the degree of difference in SmPCs for a drug)

Table 2 Categories for the second classification step (based on the impact of the SmPC discrepancy for a drug on patients)

\begin{tabular}{ll}
\hline Categories & Definition \\
\hline Critical & Any major differences in the section on Contraindications (Section 4.3) \\
& Any major differences in the section on Warnings and Precautions (Section 4.4) \\
& Not explained or compensated for elsewhere in the label \\
Major & Missing a serious event in Section 4.8 \\
& Checked IME/DME lists of severe events to be considered \\
Minor & Missing nonserious events in Section 4.8 \\
& Possible explanation: a generic company did not detect a signal that was identi- \\
& fied by the originator company \\
Very minor & The same elements are proposed in the labels but reported in different sections \\
& Safety information reported in the same section but different wording is used \\
Fully aligned & Differences in event frequencies \\
& No significant differences between the compared SmPCs
\end{tabular}

$D M E$ designated medical event, IME important medical event, SmPC summary of product characteristics

\begin{tabular}{ll}
\hline Potential impact on patients & Definition $^{\mathrm{a}}$ \\
\hline Fatal & A drug reaction directly or indirectly causing death \\
Life-threatening & A serious condition that leads to hospitalization \\
Severe & Intense reaction causing great pain, difficulty, worry, or damage \\
Medium & Moderate reactions that are distressing or intolerable for the patient \\
Minor & Mild alterations of lesser importance \\
No impact & Editorial changes \\
& The patient will not be affected \\
\hline
\end{tabular}

${ }^{\mathrm{a}}$ Adapted from [10] 


\subsection{Drug Age Versus Discrepancy Count}

To determine if the discrepancies were correlated with the length of time that the drug had been on the market, the EU reference date (EURD) list was consulted to verify the exact dates on which the 31 drugs were first marketed. The median of the EURD years for the first classification step was calculated to determine whether a longer time on the market led to greater SmPC discrepancy.

\subsection{Data Analysis}

The consistency and differences between the information presented in each individual safety-relevant section and for the full content of the compared SmPCs were analyzed. The percentile distribution of the different discrepancy classifications was calculated. The same method was applied to the potential impact on patients.

\section{Results}

\subsection{Drug Selection}

Among the 50 drugs selected initially, 31 were eligible. The distribution of these 31 drugs among the 13 ATC classes is shown in Table 6 of the Appendix. For 11 of the ineligible drugs, there was only one product with the active ingredient available on the eMC, two products had only one formulation in eMC and six products could not be compared due to different formulations (refer to Table 7 of the Appendix).

On average, more than three SmPCs were compared for each drug. For 24 of the selected drugs, an originator product was identified. For four of the drugs, there was no comparison to the originator product because either the originator product was unavailable on the $\mathrm{eMC}$ or it had a different formulation to that of the generic product. For the remaining three drugs, the originator product was not identified (refer to Table 8 of the Appendix).

\subsection{Consistency and Discrepancy}

\subsubsection{All SmPC Sections}

In the first classification step, the 31 selected drugs were assessed and assigned to the following five categories: fully aligned, critical, major, minor, and very minor (refer to Table 1). For 13 (41.9\%) of those drugs, there were critical label differences between originator and generic products; major label differences were seen for $6(19.4 \%)$ drugs, minor label differences were observed for 7 (22.6\%) drugs, and very minor differences were noted for $5(16.1 \%)$ drugs (Table 3). Over $60 \%$ of the drugs $(19,61.3 \%)$ presented important (critical, major) label differences between originator and generic products, and none of the drugs exhibited full label alignment between originator and generic products.

The median EURD was calculated for each classification to explore the potential correlation between the length of time that the drug had been on the market and the level of originator/generic label discrepancy. For drugs that presented critical label differences, the median EURD was 1969; for those with major differences, it was 1978; and for those with minor and very minor differences, the median EURD was 1980.

\subsubsection{Contraindications (Section 4.3 in the SmPC)}

Fourteen of the 31 selected drugs showed discrepancies between originator and generic products in the content of the Contraindications section of the SmPC. Of these, 10 (71.5\%) drugs were assessed as having critical differences and 4 $(28.5 \%)$ as having very minor differences in the content of the Contraindications section (Table 4). Neither major nor minor differences in the content of the Contraindications section.

\subsubsection{Warnings and Precautions (Section 4.4 in the SmPC)}

Twenty of the 31 selected drugs presented discrepancies between originator and generic products in the content of the Warnings and Precautions section of the SmPC. Of these, 11 $(55.0 \%)$ drugs were assessed as having critical differences, $4(20.0 \%)$ as having major differences, $2(10.0 \%)$ as having minor differences, and $3(15.0 \%)$ as having very minor differences in the content of the Warnings and Precautions section (Table 4). Seventy-five percent $(15 ; 75 \%)$ of the drugs presented important (critical, major) differences between originator and generic products in the content of the Warnings and Precautions section of the SmPC.

\subsubsection{Undesirable Effects (Section 4.8 in the SmPC)}

Nineteen of the 31 selected drugs presented discrepancies between originator and generic products in the content of the Undesirable Effects section of the SmPC. Of these, 6 (31.6\%)

Table 3 Classification of the discrepancy between the SmPCs of originator and generic products for all 31 selected drugs

\begin{tabular}{lcl}
\hline Classification & Number of drugs $N(\%)$ & Median EURD \\
\hline Critical & $13(41.9 \%)$ & 1969 \\
Major & $6(19.4 \%)$ & 1978 \\
Minor & $7(22.6 \%)$ & 1980 \\
Very minor & $5(16.1 \%)$ & \\
Total & $31(100 \%)$ & \\
\hline
\end{tabular}


Table 4 Classification of the discrepancy between the safety sections of the SmPCs of originator and generic products for all 31 selected drugs

\begin{tabular}{lccc}
\hline Classification & $\begin{array}{l}\text { Contraindica- } \\
\text { tions } \\
\text { Number of } \\
\text { drugs } N(\%)\end{array}$ & $\begin{array}{l}\text { Warnings and } \\
\text { Precautions } \\
\text { Number of drugs } \\
N(\%)\end{array}$ & $\begin{array}{l}\text { Undesirable } \\
\text { Effects } \\
\text { Number of } \\
\text { drugs } N(\%)\end{array}$ \\
\hline Critical & $10(71.5 \%)$ & $11(55.0 \%)$ & $6(31.6 \%)$ \\
Major & $0(0 \%)$ & $4(20.0 \%)$ & $5(26.3 \%)$ \\
Minor & $0(0 \%)$ & $2(10.0 \%)$ & $6(31.6 \%)$ \\
Very minor & $4(28.5 \%)$ & $3(15.0 \%)$ & $2(10.5 \%)$ \\
Total & $14(100 \%)$ & $20(100 \%)$ & $19(100 \%)$ \\
\hline
\end{tabular}

drugs were assessed as having critical differences, 5 (26.3\%) as having major differences, $6(31.6 \%)$ as having minor differences, and $2(10.5 \%)$ as having very minor differences in the content of the Undesirable Effects section (Table 4). Over $55 \%(11,57.9 \%)$ of the drugs exhibited important (critical, major) differences between originator and generic products in the content of the Undesirable Effects section.

\subsubsection{Overdose (Section 4.9 in the SmPC)}

Minor to major differences between originator and generic products in the content of the Overdose section of the SmPC were seen for five drugs. No critical discrepancies between originator and generic products in the content of this section of the SmPC were observed.

\subsection{Impact on Patients}

Labeling misalignment between originator and generic products that could have a fatal or life-threatening impact, a severe impact, a medium impact, and a minor impact or no impact on the patient was seen for $4(12.9 \%), 11(35.5 \%)$, $6(19.35 \%)$, and $10(32.25 \%)$ of the drugs (Table 5). For almost half $(15,48.4 \%)$ of the drugs, labeling misalignment between originator and generic products had the potential to have a critical (fatal, life-threatening, or severe) impact on the patient.

\subsection{Literature Search}

Of the 50 events that were found to be inconsistently mentioned in the SmPCs considered in this analysis, 26 were identified in articles published in the scientific literature. The OCEBM level of evidence for each article ranged from 1 to 5 , with a median value of 4 . The Micromedex [12] entry for each drug was also examined to check if they mentioned the discrepant events that were noted in the SmPCs. Four of the discrepant events were mentioned in the Micromedex entries of the respective drugs.
Table 5 Classification of the potential impact on patients of label discrepancies between originator and generic products for all 31 selected drugs

\begin{tabular}{lc}
\hline Potential impact on patients & Number of drugs $N(\%)$ \\
\hline Fatal/life-threatening & $4(12.90 \%)$ \\
Severe & $11(35.50 \%)$ \\
Medium & $6(19.35 \%)$ \\
Minor/no impact & $10(32.25 \%)$ \\
Total & $31(100 \%)$ \\
\hline
\end{tabular}

\section{Discussion}

Our study has identified that major discrepancies in pharmaceutical product labeling — sometimes involving a lack of notification of life-threatening side effects or contraindications - are a reality.

The first finding was that all 31 of the drugs considered in this work presented misalignment between the SmPCs of originator and generic products; indeed, critical discrepancies between SmPCs were observed for 13 of those drugs. Beyond these critical discrepancies, the potential impact of the differences between SmPCs of originator and generic products varied substantially, with six drugs presenting label discrepancies that could have a major impact on patients, seven presenting label discrepancies that could have a minor impact, and five presenting label discrepancies that could have a very minor impact on patients.

In terms of discrepancies between originator and generic products in particular sections of the SmPC, there were 10 critical discrepancies in the Contraindications section and 11 crucial discrepancies in the Warnings and Precautions section. These discrepancies are particularly striking, as they place specific subpopulations, such as young children and comorbid patients, at high risk.

Four of the SmPC discrepancies had the potential to be life-threatening, or even to lead to the death of the patient, which is of great concern. Among the potentially fatal discrepancies, one concerned adrenaline: a contraindication for patients with underlying ventricular fibrillation, cardiac dilatation, or coronary insufficiency was missing from a SmPC for a generic product. This discrepancy could lead to ventricular arrhythmias and/or coronary ischemias in such patients. The other potentially fatal discrepancy concerned promethazine: a contraindication for patients aged 2 years or less was missing from the generic product labels, leading to a risk of respiratory depression in this subpopulation. These represent crucially important patient safety omissions from the labels.

A literature search was performed to check if the discrepant events in the SmPCs were supported by scientific publications. The resulting relatively low EBM score of 4 can 
be explained by the fact that many of the articles reviewed were individual case reports or case series. Only three discrepancies were found to have a level of evidence of 1 or 2. Of the 50 discrepant events in SmPCs identified in this work, half were supported or at the very least identified in case reports, case series, randomized clinical trials, cohort studies, or Micromedex entries. This suggests that if MAHs do not continuously compare labels with their competitors (generic or originator companies), the publicly available scientific information will not be updated to include new side effects. Moreover, this crucial information will not be available to patients and physicians. This fact may lead to a lack of awareness by patients and physicians of potentially important safety information.

Often, the lack of a complete set of available data, including clinical development and large database, may also explain why signal detection is less efficient for generic manufacturers.

We assumed that the longer an originator product had been on the market, the more discrepancies there would be between its label and those of corresponding generic products. Indeed, this appeared to be the case for critical label discrepancies. As mentioned above, the SmPC for a product should be continually updated. Thus, in the absence of constant and systematic comparisons with the SmPCs for other products containing the same active ingredient, inconsistencies between the SmPCs of those products will accumulate over time.

There are a number of reasons for the observed discrepancies between the labels of originator and generic products. For instance, it is expensive to update the SmPC, so many pharmaceutical companies will tend to wait until it is necessary to update several SmPCs and then submit several modifications within one variation package. The wealth of products available on the market means that regulators cannot closely monitor all marketed products for potential discrepancies, and there is no clear legislation ensuring that MAHs perform label comparisons and updates on a systematic basis. Health authorities can force pharmaceutical companies to quickly modify SmPCs, but this is usually done during events of public health concern or if the drug is linked to severe (e.g., life-threatening) events. Public awareness of a particular safety issue with a medicinal product can also pressure its manufacturer to keep the label of the product up to date. It should also be stressed that in the absence of any health authority request for a label update, MAHs do not incur any financial penalty for not keeping product labels up to date.

This study has several limitations. Only publicly available SmPCs were compared, the study focused on just one country (the UK), and many of the drugs chosen had a limited number of different products in the eMC, meaning that much of the available data were associated with just a few of the drugs considered. This study was also unable to elucidate the regulatory reasons behind the observed discrepancies. Finally, the originator products for some of the drugs considered here were sometimes challenging to identify because the drugs had been on the market for a long time, leading the authors to use the SmPC for the product that was first to the market for baseline comparison.

\section{Conclusions}

Despite its limitations, this study demonstrated that-for the sample of pharmaceutical products considered hereSmPC alignment between established generic and originator medicinal products is inefficient, and may lead to the diffusion of discrepant messages about a medication to healthcare professionals and patients.

Other studies have identified differences between countries in the safety information provided for the same medication or class of treatment [13-17]. However, this study investigated the differences between the safety information on product labels provided by different MAHs using a qualitative approach and focusing on the potential impact on patient health. Further and broader investigations into product labeling will be needed to confirm and expand on these results. Given the drug registration process and regulations in force in Europe, it is likely that this nonalignment of safety information provided on product labels will occur less frequently in the future. Nevertheless, to address this lack of label alignment, health authorities such as the EMA and the FDA must conduct retrospective analyses of all drugs on the market. These analyses could be performed during the evaluation of aggregate reports.

\section{Compliance with Ethical Standards}

Funding No funding was used to assist in the preparation of this article.

Conflict of interest Alexandra Thoenes (M.D.), Luca Cariolato (Ph.D.), and Alexis Pinçon (M.D., M.Sc.) are paid employees of United BioSource Corporation (UBC), Switzerland, where they work as a project physician, senior drug safety scientist, and director of global safety writing and medical services, respectively. Julian Spierings (M.Sc.) is a paid employee of Vifor Pharma, Switzerland, where he is the head of medical writing.

Data sharing The datasets generated and/or analyzed during the current study are available from the corresponding author on reasonable request.

Open Access This article is licensed under a Creative Commons Attribution-NonCommercial 4.0 International License, which permits any non-commercial use, sharing, adaptation, distribution and reproduction in any medium or format, as long as you give appropriate credit to the original author(s) and the source, provide a link to the Creative 
Commons licence, and indicate if changes were made. The images or other third party material in this article are included in the article's Creative Commons licence, unless indicated otherwise in a credit line to the material. If material is not included in the article's Creative Commons licence and your intended use is not permitted by statutory regulation or exceeds the permitted use, you will need to obtain permission directly from the copyright holder.To view a copy of this licence, visit http://creativecommons.org/licenses/by-nc/4.0/.

\section{Appendix}

See Tables 6, 7, and 8 .
Table 6 Drugs stratified by ATC classification

\begin{tabular}{llr}
\hline ATC code & Anatomical group & $\begin{array}{r}\text { Number } \\
\text { of drugs }\end{array}$ \\
\hline A & Alimentary tract and metabolism & 2 \\
B & Blood and blood-forming organs & 2 \\
C & Cardiovascular system & 10 \\
G & Genitourinary system and reproductive hormones & 2 \\
J & Anti-infectives for systemic use & 2 \\
L & Antineoplastic and immunomodulating agents & 1 \\
M & Musculoskeletal system & 1 \\
N & Nervous system & 7 \\
R & Respiratory system & 4 \\
\hline
\end{tabular}

ATC Anatomical Therapeutic Chemical

Ineligible drugs

Alprazolam

Calcium chloride

Captopril

Captopril/hydrochlorothiazide

Dacarbazine

Doxorubicin hydrochloride

Ergocalciferol

Ethinylestradiol

Hydroxyzine

Isosorbide dinitrate
Lidocaine

Lorazepam

Meclozine

Metolazone

Naloxone

Phenoxymethylpenicillin

Piracetam

Sodium oxybate

Teicoplanin 
Table 8 Summary of the data for the drugs selected for analysis in this study

\begin{tabular}{|c|c|c|c|c|c|c|c|c|}
\hline Product & EURD & $\begin{array}{l}\text { First clas- } \\
\text { sification }\end{array}$ & Originator & $\begin{array}{l}\text { ATC } \\
\text { class }\end{array}$ & $\begin{array}{l}\text { Impact on } \\
\text { patients (2nd } \\
\text { classification) }\end{array}$ & $\begin{array}{l}\text { SmPC } \\
\text { section(s) }\end{array}$ & $\begin{array}{l}\text { Number of } \\
\text { companies } \\
\text { compared }\end{array}$ & $\begin{array}{l}\text { Literature } \\
\text { EBM }\end{array}$ \\
\hline $\begin{array}{l}\text { Adrenaline } \\
\text { (epinephrini } \\
\text { hydrochloridum) }\end{array}$ & $15 / 05 / 1952$ & Critical & Not known & $\mathrm{C}$ & Fatal & 4.3 & 2 & EBM 4-5 \\
\hline Amphotericin B & $19 / 03 / 1958$ & Critical & BMS (compared) & $\mathrm{J}$ & Severe & $4.4,4.8$ & 3 & EBM 4 \\
\hline $\begin{array}{l}\text { Beclomethasone } \\
\text { dipropionate }\end{array}$ & $13 / 12 / 1966$ & Critical & GSK (compared) & $\mathrm{R}$ & Life-threatening & $4.4,4.3$ & 2 & EBM 3a \\
\hline Cilostazol & Not available & Very minor & Otsuka (compared) & B & Medium & $4.3,4.4$ & 4 & EBM 0 \\
\hline $\begin{array}{l}\text { Codeine monohy- } \\
\text { drate }\end{array}$ & 06/09/1954 & Critical & $\begin{array}{l}\text { Boots/Bristol (not com- } \\
\text { pared) }\end{array}$ & $\mathrm{N}$ & Severe & 4.3 & 4 & EBM 0-3a \\
\hline $\begin{array}{l}\text { Diltiazem hydro- } \\
\text { chloride }\end{array}$ & 02/05/1979 & Critical & $\begin{array}{l}\text { Aventis (not compared; } \\
\text { compared to Sanofi) }\end{array}$ & $\mathrm{C}$ & Severe & $4.3,4.4$ & 3 & EBM 4 \\
\hline Alprostadil & 23/07/1981 & Critical & Pfizer (compared) & $\mathrm{G}$ & Severe & $4.4,4.8$ & 2 & EBM $0-2 b$ \\
\hline Colistimethate & 08/10/1962 & Minor & Teva (compared) & $\mathrm{J}$ & Minor & $4.8,4.9$ & 3 & NA \\
\hline Salbutamol & $31 / 01 / 1969$ & Major & GSK (compared) & $\mathrm{R}$ & Medium & $4.4,4.8,4.9$ & 3 & NA \\
\hline Methylphenidate & $06 / 10 / 1954$ & Major & Novartis (compared) & $\mathrm{N}$ & Severe & 4.4 & 3 & EBM0 \\
\hline Docusate sodium & 01/01/1976 & Critical & Not known & A & Medium & $4.3,4.4$ & 2 & EBM0 \\
\hline Levocetirizine & 03/01/2001 & Major & UCB (compared) & $\mathrm{R}$ & Medium & $\begin{array}{l}4.4,4.6,4.8 \\
4.9\end{array}$ & 4 & EBM 0 \\
\hline Cetirizine & $06 / 11 / 1986$ & Major & UCB (compared) & $\mathrm{R}$ & Severe & $4.8,4.4$ & 5 & EBM 4 \\
\hline Indapamide & $25 / 02 / 2002$ & Major & Servier (compared) & $\mathrm{C}$ & Medium & 4.8 & 5 & EBM 4 \\
\hline $\begin{array}{l}\text { Isosorbide mon- } \\
\text { onitrate }\end{array}$ & $01 / 05 / 1980$ & Minor & $\begin{array}{l}\text { Topridge/Merus (com- } \\
\text { pared to Merus) }\end{array}$ & $\mathrm{C}$ & No impact & 4.8 & 4 & NA \\
\hline Azathioprine & $13 / 12 / 1965$ & Minor & Aspen (compared) & $\mathrm{L}$ & Minor & 4.8 & 4 & NA \\
\hline Desogestrel & 01/07/1998 & Very minor & MSD (compared) & G & Minor & 4.3 & 5 & NA \\
\hline Atorvastatin & 07/11/1996 & Critical & Pfizer (compared) & $\mathrm{C}$ & Severe & $4.3,4.4,4.8$ & 3 & EBM 1B \\
\hline Nifedipine & $31 / 08 / 1993$ & Critical & Bayer (compared) & $\mathrm{C}$ & Severe & $4.3,4.4,4.8$ & 3 & EBM 0-4 \\
\hline Furosemide & $01 / 01 / 1955$ & Major & $\begin{array}{l}\text { Aventis (on eMC, but } \\
\text { different formulation) }\end{array}$ & $\mathrm{C}$ & Severe & 4.8 & 4 & EBM 4 \\
\hline Penicillamine & $04 / 04 / 1963$ & Minor & Alliance (compared) & M & Minor & $4.4,4.8$ & 3 & NA \\
\hline Atenolol & $19 / 02 / 1976$ & Critical & $\begin{array}{l}\text { AstraZeneca (not com- } \\
\text { pared, not on eMC) }\end{array}$ & $\mathrm{C}$ & Severe & $4.3,4.4$ & 2 & EBM $2 A-4$ \\
\hline Omeprazole & $15 / 04 / 1987$ & Minor & AstraZeneca (compared) & A & Medium & 4.8 & 4 & EBM 4 \\
\hline Amiodarone & $20 / 12 / 1966$ & Very minor & Zentiva (compared) & $\mathrm{C}$ & Minor & $\begin{array}{l}4.3,4.4,4.8 \\
\quad 4.9\end{array}$ & 3 & NA \\
\hline Promethazine & $26 / 10 / 1948$ & Critical & Not known & $\mathrm{N}$ & Fatal & $4.3,4.4$ & 2 & EBM 0 \\
\hline Atropine sulfate & $31 / 08 / 1945$ & Critical & Martindale (compared) & $\mathrm{C}$ & Life-threatening & $4.4,4.8,4.3$ & 2 & EBM 0 \\
\hline Urokinase & $24 / 05 / 1972$ & Minor & $\begin{array}{l}\text { Syner-Medica (com- } \\
\text { pared) }\end{array}$ & B & No impact & $\begin{array}{l}\text { Editorial } \\
\text { only }\end{array}$ & 2 & NA \\
\hline Venlafaxine & $23 / 09 / 1993$ & Minor & Pfizer (compared) & $\mathrm{N}$ & Minor & $4.4,4.8$ & 2 & NA \\
\hline Tramadol & $18 / 04 / 1973$ & Critical & Grunenthal (compared) & $\mathrm{N}$ & Severe & $\begin{array}{l}4.3,4.4,4.8 \\
\quad 4.9\end{array}$ & 3 & EBM $3 \mathrm{~A}-5$ \\
\hline Duloxetine & $11 / 08 / 2004$ & Very minor & Eli Lilly (compared) & $\mathrm{N}$ & No impact & 4.8 & 4 & NA \\
\hline Escitalopram & $31 / 12 / 2001$ & Very minor & Lundbeck (compared) & $\mathrm{N}$ & No impact & $4.3,4.4$ & 3 & NA \\
\hline
\end{tabular}

ATC Anatomical Therapeutic Chemical, EBM evidence-based medicine, EURD EU reference date, NA not applicable, SmPC summary of product characteristics 


\section{References}

1. Duke J, Friedlin J, Ryan P. A quantitative analysis of adverse events and "overwarning" in drug labeling. Arch Int Med. 2011;171:941-54

2. European Medicines Agency. Scientific guidelines with SmPC recommendations, rev 4, 25 January 2017. London: EMA; 2017.

3. King JP, Davis TC, Bailey SC, Jacobson KL, Hedlund LA, Di Francesco L, Parker RM, Wolf MS. Developing consumercentered, nonprescription drug labeling. Am J Prev Med. 2016;40:593-8.

4. Kircik L, Sung JC, Stein-Gold L, Goldenberg G. United States Food and Drug Administration product label changes. J Clin Aesthet Dermatol. 2016;9:39.

5. Marcussen M. Branded versus generic label alignment: assessing safety and compliance in the US. PharmacoVigilance Rev 2016;9(1):6-10.

6. European Commission. Directive 2004/27/EC of the European Parliament and of the Council of 31 March 2004 amending Directive 2001/83/EC on the community code relating to medicinal products for human use. 2004. https://ec.europa.eu/health/sites/ health/files/files/eudralex/vol-1/dir_2004_27/dir_2004_27_en.pdf. Accessed Feb 2019.

7. European Commission. A guideline on summary of product characteristics revision 2. Brussels: EC; 2009.

8. DataPharm Ltd. The electronic Medicines Compendium. Leatherhead: DataPharm Ltd.; 2019. https://www.medicines.org.uk/emc. Accessed Feb 2019.

9. WHO Collaborating Centre for Drug Statistics Methodology. ATC classification. Oslo: WHO Collaborating Centre for Drug Statistics Methodology; 2012.
10. Merck and Co., Inc.. Merck Manuals: severity of adverse drug reactions. https://www.merckmanuals.com. Accessed Feb 2019.

11. Oxford Center for Evidence-Based Medicine. Classification. Oxford: OCEBM; 2009. https://www.cebm.net/2009/06/oxfor d-centre-evidence-based-medicine-levels-evidence-march-2009/. Accessed June 2019.

12. IBM Corp. Micromedex. https://www.micromedexsolutions.com/ micromedex2/librarian/ss1/true. Accessed June 2019.

13. Alshammari TM, Devadasu VR, Rathnam RP. Comparison of the safety information on drug labels in three developed countries: the USA, UK and Canada. Saudi Pharm J. 2017;25(8):1103-7.

14. Eriksson R, Aagaard L, Jensen LJ, Borisova L, Hørlück D, Brunak $\mathrm{S}$, Hansen EH. Discrepancies in listed adverse drug reactions in pharmaceutical product information supplied by the regulatory authorities in Denmark and the USA. Pharmacol Res Perspect. 2014;2(3):e00038.

15. Nieminen O, Kurki P, Nordström K. Differences in product information of biopharmaceuticals in the EU and the USA: implications for product development. Eur J Pharm Biopharm. 200560(3):319-26.

16. Duke J, Friedlin J, Li X. Consistency in the safety labeling of bioequivalent medications. Pharmacoepidemiol Drug Saf. 2013;22:294-301.

17. Shimazawa R, Kano Y, Ikeda M. Natural language processingbased assessment of consistency in summaries of product characteristics of generic antimicrobials. Pharmacol Res Perspect. 2018;6:e0435 (ORCID: 0000-0001-5157-1362). 